\title{
Uterine Tumor Resembling Ovarian Sex Cord Tumor: Clinicopathological Characteristics of a Rare Case
}

\author{
Chu Van Nguyen a, buyen Thi Phung ${ }^{a}$ Luan Thi Dao ${ }^{b}$ \\ Dang Hong Hai Tab Minh Ngoc Tran ${ }^{b}$ \\ aDepartment of Quan Su Pathology, National Cancer Hospital, Hanoi, Vietnam; \\ bHanoi Medical University, Hanoi, Vietnam
}

\section{Keywords}

Uterine tumor resembling ovarian sex cord tumor - Clinical characteristics · Pathology

\begin{abstract}
Uterine tumor resembling ovarian sex cord tumor (UTROSCT) is a rare indolent stromal neoplasm of unclear histogenesis with a distinct histopathological entity. Immunophenotypes of sex cord positivity are the most significant information to confirm the diagnosis. We present the case of a 61-year-old female with a polypoid mass in the uterus which was successfully removed by surgical resection as hysterectomy. The pathological diagnosis was UTROSCT, which was characterized microscopically by sex cord images and immunohistochemical features of calretinin, CD99, and WT1 positivity.

\section{Introduction}

From a pathological point of view, uterine tumor resembling ovarian sex cord tumor (UTROSCT) is very similar to some ovarian sex cord tumors, which are a rare type of stromal tumor. Initially, in 1945, it was described by Morehead and Bowman [1]. Subsequently, Clement and Scully [2] conducted a study on 14 such cases and further classified the neoplasms into two distinct subgroups based on clinicopathological features in 1976. To date, only 82 cases have been reported in the English-language literature [3-8].

Here, we present the clinicopathological characteristics of the first case in Vietnam and compare it with the related literature to raise the awareness of this tumor. 
Nguyen et al.: UTROSCT: Clinicopathological Characteristics of a Rare Case

\section{Case Presentation}

\section{Clinical Characteristics}

The patient was a 61-year-old postmenopausal woman who was admitted to Hanoi Medical University Hospital, Vietnam, at the beginning of March 2020. She presented with irregular, abnormal vaginal bleeding which had lasted for 2 days. The histopathological results of an endometrial curettage and a Pap smear were not abnormal.

Transvaginal ultrasonography demonstrated a mass with a heterogeneous echo that was located in the lumen, with partial invasion of the uterine wall. Some of the bilateral pelvic lymph nodes were observed to be $<10 \mathrm{~mm}$ in size. The paraaortic lymph node was not found. MRI at $1.5 \mathrm{~T}$ was performed, which revealed a solid neoplasm $53 \mathrm{~mm}$ in diameter in the lumen.

The patient was diagnosed with suspicion for endometrioid cancer, FIGO stage IB. She accepted and underwent an extended laparoscopic hysterectomy with bilateral pelvic lymph node dissection. Additionally, she received anti-inflammatory, rehydration, and conventional anticoagulation therapy after the surgery. After 1 month, the patient improved and was recovered.

\section{Pathological Features}

Macroscopic Characteristics

On macroscopic examination, the specimen was grossly uterine. In the lumen, a polypoid mass, originating from the lateral wall nearby the fundal area, was seen that occupied about half of the muscle thickness. The endometrium was smooth and thin. On section, the tumor had a moderate margin, solid with a small cystic structure, $53 \times 40 \times 35 \mathrm{~mm}$ in size. The cut surface was soft and appeared pale yellow in color. Necrosis and hemorrhage were not observed.

\section{Microscopical Features}

Hematoxylin and eosin-stained slides were examined. Some microcysts and small foci of lipidized cells were mixed in the solid tumor areas (Fig. 1). The tumor tissue was underlaying the benign, thin, intact endometrium. The tumor architecture displayed various patterns, including anastomosing cords of 1-2 cells width, broad trabeculae, small nests, micropapillary-like (Fig. 2), sertoliform or retiform tubular structures, and diffuse sheets of epithelioid cells. The neoplastic cells were quite atypical with round- to oval-shaped little pleomorphic, eccentric nuclei with a clear nuclear membrane, and inconspicuous nucleoli. Sometimes, the tumor cells were spindle-shaped. Mitosis was not observed. The tumor cytoplasm was scant, indistinct, and eosinophilic. The microscopical images looked like ovarian Sertoli cell tumor. The tumor boundary and surrounding tissue were unclear, as both the myometrium and the endometrium were affected by tumor invasion. The intervening stroma was sparse and showed hyalinization with small scattered lymphocytic foci (Fig. 1).

All bilateral pelvic lymph nodes, including 4 right nodes and 9 left nodes, were negative.

Immunohistochemical Findings

Immunohistochemical (IHC) evaluation revealed strong positivity for calretinin (Fig. 3) and estrogen receptor, as well as focal expression of melan-A and CD99. WT1 (Wilm's tumor protein 1) was expressed at a faint level. Positive Ki-67 was counted at $<5 \%$. The tumor cells displayed EMA negativity.

The above pathological and IHC findings confirmed that this tumor was consistent with a UTROSCT. 


\section{Case Reports in Oncology}

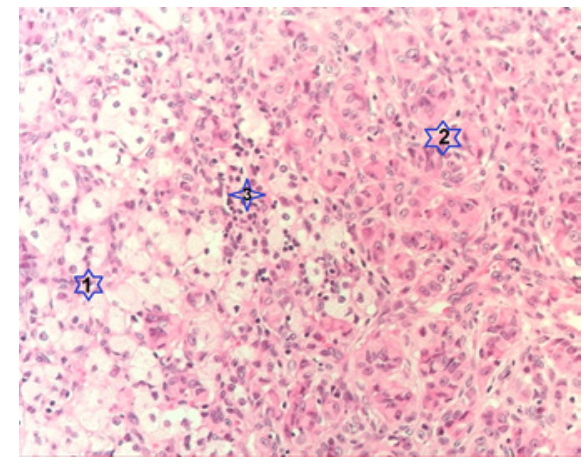

Fig. 1. Microscopic picture showing the foci of lipidized tumor cells (1), broad trabecular and small nest areas (2), and the scattered lymphocytic stroma (3). HE. $\times 20$.

Fig. 3. Photomicrograph indicating strong positivity for calretinin in tumor cells, with a microcyst architecture (1). IHC stain. $\times 20$.

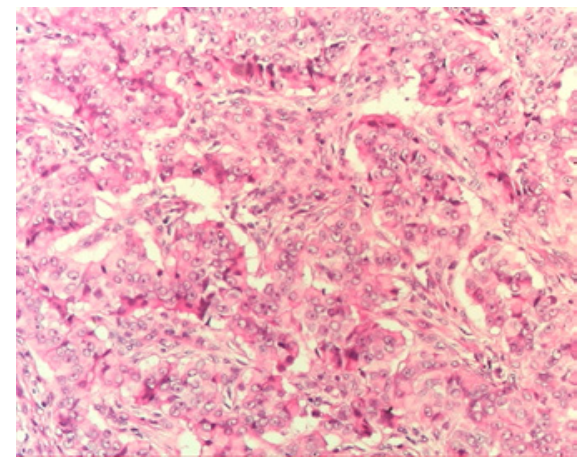

Fig. 2. Image presenting micropapillarylike structures of epithelioid tumor cells. HE. $\times 20$. All bilateral pelvic lymph nodes, including 4 right nodes and 9 left nodes, were negative.

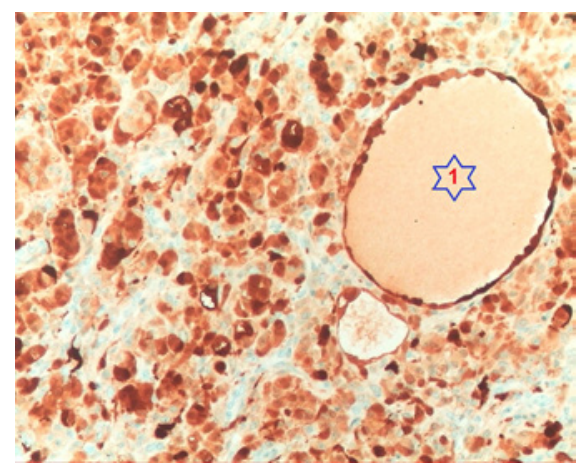

\section{Discussion}

UTROSCT is a rare stromal neoplasm of unknown etiology. This tumor is an indolent entity. The neoplasm is seen in premenopausal or postmenopausal females, and the medium age is 45 years. Patients typically present with vaginal bleeding or lower abdominal discomfort or pain, though many patients do not display any symptoms [5]. The tumor may be located in the subendometrial or intramyometrial tissue, or in the subserosa. Generally, UTROSCT reveals expanded growth with or without invasion of the surrounding tissue [3].

In 1976, Clement and Scully [2] divided these neoplasms into two groups. The first group (type I) has histopathological characteristics similar to those of endometrial stromal tumors, with $10-40 \%$ areas of sex cord-like architecture. This category is associated with a metastasis or recurrence risk, and is named endometrial stromal tumor with sex cord-like elements (ESTSCLE). In contrast, the second group (type II) is named UTROSCT, including $>50 \%$ sex cord-like structures and behaving in a benign fashion [2]. UTROSCTs are usually confirmed by IHC expression of sex cord positivity [9], and they are classified as a miscellaneous group of uterus tumors in the WHO 2014 classification. In ESTSCLE, fusion of two novel genes UAZF1 and JJAZ1) occurs, which is not observed in UTROSCT, emphasizing that UTROSCT is another entity than ESTSCLE; nevertheless, the origin of UTROSCT remains unknown [10]. The above clinicopathological features are considered to be diagnostic criteria for separating between the two neoplastic types.

In the current presentation, the microscopical features resembled ovarian Sertoli cell tumor. The IHC findings demonstrated these characteristics. The histopathological features 
and IHC expression may be considered to be those of ovarian Sertoli cell tumor. Actually, the group of ovarian sex cord stromal tumors is composed of many tumor types which follow various aggressive clinical courses. Consequentially, it may be essential whether UTROSCT should be categorized among different histopathological types that are similar in morphology to ovarian sex cord stromal tumors. In the future, further analyses should be conducted for a better evaluation of UTROSCT with different subtypes.

Since there are virtually no specific histopathological characteristics, the diagnosis of UTROSCT is made exclusively based on morphologic examination. An array of architectural patterns is valuable for suggesting UTROSCT; they include epithelioid cells, as well as nests of sex cord-like components forming in plexiform cords, a watered-silk pattern, microcysts, and diffuse patterns of growth $[9,11]$. These findings suggest that UTROSCT may result from divergent differentiation in endometrial stromal tumors or represent a distinct group of uterine tumors with sex cord-like differentiation that is closer in histogenesis to ovarian sex cord stromal tumors [11]. Recently conducted studies have illustrated that the immunophenotypes of sex cord positivity convey the most significant information. It is a panel of five IHC markers, including calretinin, inhibin, CD99, melan-A, and WT1 [9, 12]. Positivity for calretinin, and for at least one more of the other abovementioned markers, is necessary in order to obtain the diagnosis of UTROSCT $[2-4,7,8,13]$. On the other hand, endometrial stromal tumors with sex cord-like elements usually express only one of these sex cord IHC markers, mostly calretinin [13]. In the present case, immunohistochemically, the tumor cells were positive for four of these five IHC markers, i.e., calretinin, melan-A, WT1, and CD99, which confirms the diagnosis. The findings are consistent with those of the study by Sadeh et al. [7].

Although UTROSCT is a distinct histopathological entity, numerous benign tumors and cancers can cause a diagnostic pitfall. UTROSCTs have to be separated into the different tumors such as epithelioid leiomyoma, low-grade endometrial stromal sarcoma with sex cord elements, endometrioid carcinoma with sex cord-like features, adenosarcoma, and carcinosarcoma [9]. Of course, ESTSCLEs need to be differentiated from UTROSCTs, owing to their more aggressive course and metastatic and recurrent capabilities [9]. The current case was differentiated from carcinosarcoma on microscopic examination.

These neoplasms present with an indolent clinical course and thus only call for a more conservative and less invasive treatment decision [9]. Hysterectomy with bilateral salpingooophorectomy or hysteroscopic tumor resection are considered suitable treatment options; nevertheless, the literature regarding disease management is limited [14]. Sometimes, it is necessary to dissect regional lymph nodes such as pelvic and/or para-aortic lymph nodes. In the present case, the bilateral pelvic lymph nodes were removed. Fortunately, all of them were negative. UTROSCT is considered having a low malignant potential, with occasional extrauterine or lymph node metastasis or recurrence risk [12]. Previous studies have reported extrauterine or lymph node metastasis $[12,14,15]$. It is essential that patients with UTROSCT be closely followed up to prevent these events, due to the unknown tumor characteristics.

\section{Conclusion}

The present case suggests that it is crucial for pathologists to recognize this rare entity. Diagnostically, it has to be differentiated from other lesions, particularly tumors with intermediate malignant potential. 


\section{Case Reports in Oncology}

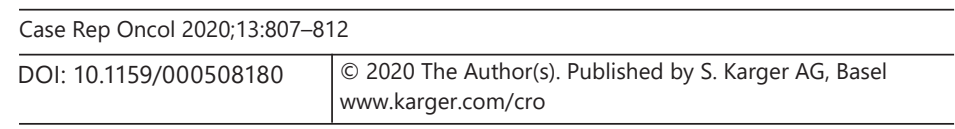

Nguyen et al.: UTROSCT: Clinicopathological Characteristics of a Rare Case

\section{Acknowledgments}

The authors would like to thank Prof. Tran Van Thuan, MD, PhD, who is the Director of the National Cancer Hospital and National Cancer Institute, Hanoi, Vietnam, and the Oncology Department of Hanoi Medical University Hospital for their assistance and support with this report.

\section{Statement of Ethics}

Informed consent was obtained from the patient for the presentation of her case along with the associated microscopical images.

\section{Conflict of Interest Statement}

The authors declare no potential conflicts of interest with respect to the research, authorship, and/or publication of this article.

\section{Funding Sources}

The authors received no financial support for the research, authorship, and/or publication of this article.

\section{Author Contributions}

C.V. Nguyen participated directly in the diagnosis and follow-up of the patient, took microscopical pictures, performed the literature review, assisted in drafting of the components of the case report, and assisted in formatting the presented material. H.T. Phung performed the literature review and assisted in drafting of the components of the case report. L.T. Dao and M.N. Tran took part in diagnostic consultation and the follow-up of the patient and assisted in the literature review. D.H.H. Ta performed the HE stains and IHC staining for all presented materials.

\section{References}

1 Morehead RP, Bowman MC. Heterologous mesodermal tumors of the uterus: report of a neoplasm resembling a granulosa cell tumor. Am J Pathol. 1945;21(1):53-61.

2 Clement PB, Scully RE. Uterine tumors resembling ovarian sex-cord tumors. A clinicopathologic analysis of fourteen cases. Am J Clin Pathol. 1976;66(3):512-25.

3 Cetinkaya N, Bas S, Cuylan ZF, Erdem O, Erkaya S, Gungor T. Uterine tumors resembling ovarian sex cord tumors: a case report and literature review. Oncol Lett. 2016;11(2):1496-8.

4 Dickson BC, Childs TJ, Colgan TJ, Sung YS, Swanson D, Zhang L, et al. Uterine tumor resembling ovarian sex cord tumor: a distinct entity characterized by recurrent NCOA2/3 gene fusions. Am J Surg Pathol. 2019;43(2): 178-86.

5 Fan LL, Shen Y, Chanda K, Ren ML. Uterine tumors resembling ovarian sex-cord tumor: a case report and literature review. J Can Res Ther. 2018;14(Suppl):S1209-12.

6 Hashmi AA, Faridi N, Edhi MM, Khan M. Uterine tumor resembling ovarian sex cord tumor (UTROSCT), case report with literature review. Int Arch Med. 2014:7(1):47.

7 Sadeh R, Segev Y, Schmidt M, Schendler J, Baruch T, Lavie O. Uterine tumors resembling ovarian sex cord tumors: case report of rare pathological and clinical entity. Case Rep Obstet Gynecol. 2017;2017:2736710. 
8 Zhang X, Zou S, Gao B, Qu W. Uterine tumor resembling ovarian sex cord tumor: a clinicopathological and immunohistochemical analysis of two cases and a literature review. J Int Med Res. 2019;47(3):1339-47.

9 Pradhan D, Mohanty SK. Uterine tumors resembling ovarian sex cord tumors. Arch Pathol Lab Med. 2013; 137(12):1832-6.

10 Hermsen B, Bogliatto F, Bleeker M, Leidi L, Trum H, Comello E, et al. Uterine tumour resembling ovarian sex cord tumour (UTROSCT): experience with a rare disease. Two case reports and overview of the literature. Obstet Gynecol Cases Rev. 2015;2(4):049.

11 Gupta M, de Leval L, Selig M, Oliva E, Nielsen GP. Uterine tumors resembling ovarian sex cord tumors: an ultrastructural analysis of 13 cases. Ultrastruct Pathol. 2010;34(1):16-24.

12 Umeda S, Tateno M, Miyagi E, Sakurai K, Tanaka R, Tateishi Y, et al. Uterine tumors resembling ovarian sex cord tumors (UTROSCT) with metastasis: clinicopathological study of two cases. Int J Clin Exp Pathol. 2014; $7(3): 1051-9$.

13 Blake EA, Sheridan TB, Wang KL, Takiuchi T, Kodama M, Sawada K, et al. Clinical characteristics and outcomes of uterine tumors resembling ovarian sex-cord tumors (UTROSCT): a systematic review of literature. Eur J Obstet Gynecol Reprod Biol. 2014;181:163-70.

14 Liu CY, Shen Y, Zhao JG, Qu PP. Clinical experience of uterine tumors resembling ovarian sex cord tumors: a clinicopathological analysis of 6 cases. Int J Clin Exp Pathol. 2015;8(4):4158-64.

15 Biermann K, Heukamp LC, Büttner R, Zhou H. Uterine tumor resembling an ovarian sex cord tumor associated with metastasis. Int J Gynecol Pathol. 2008;27(1):58-60. 